\title{
Bit Error Rate analysis using Converged Welch's Method for Energy Detection Spectrum Sensing in Cognitive Radio
}

\author{
Amrit Mukherjee ${ }^{1, *}$ and Amlan Datta ${ }^{2}$ \\ ${ }^{1}$ Department of Electronics and Communication Engineering, $K$ L University, India \\ ${ }^{2}$ School of Electronics Engineering KIIT University, India
}

Received 8 June 2016; Accepted 1 November 2016

\begin{abstract}
In this Letter, a novel approach is used for improving the Bit Error Rate (BER) for non-stationary environment based spectrum sensing. Here, we have considered Converged Welch's Power Spectral Density (PSD) for analyzing of power. The signal of interest is thus further studied in terms of BER keeping the antenna and channel parameters constant. The proposed method yields an improvement of $34.6 \%$ as compared with the conventional method.
\end{abstract}

Keywords: Cognitive radio, Energy detection spectrum sensing, Welches power spectral density, Bit error rate, spectrum sensing

\section{Introduction}

The principal of Cognitive Radio (CR) is based on dynamic spectrum access model which is the most suitable and available to utilize spectrum efficiently. The problem of BER in case of CR arise because of the high data rates application based communication. Different optimizing techniques has been implemented for reducing BER with the price of sacrificing system simplicity and operating time. The implementation of Welch's method shows better results as compared to conventional Periodogram [1-5]. The convergence of the Welch's PSD is based on the converging nature of the coefficients of Welch's PSD,taking into considerations the assumptions. The proposed Converged Welch's Power Spectral (CWPS) reduces the BER with an extent of $34.6 \%$ from that of the conventional method. The novelty of the method lies in the designing and assumption that the total energy remains constant for a given real time signal. Furthermore the increase in power resolution is based on a fixed effective radius of the network.

\subsection{Effect of Noise Uncertainty}

Noise has a significant role in most communication systems and it is basically the collection of various independent sources in which thermal noise and interferences due to nearby unintended emissions are also included. So, it is standard to consider the noise as a Gaussian random process. Further, for thepossibility of the change of noise variance over time, exact estimation of noise is not possible. In the below section it is shown that the detection threshold $\lambda$ is proportional to noise variance $\sigma_{w}^{2}$. In most practical scenarios, $\sigma_{w}^{2}$ (and $\lambda$ consequently) would need to be estimated at the receiver [6].

\footnotetext{
*E-mail address: amrit1460@gmail.com

ISSN: $1791-2377$ @ 2016 Eastern Macedonia and Thrace Institute of Technology. All rights reserved.
}

The estimates are denoted by $\hat{\sigma}_{w}^{2}$ and $\lambda$. Assuming the error in estimating $\sigma_{w}^{2}$ is assumed to be as:

$$
\begin{aligned}
& \left(1-\varepsilon_{1}\right) \sigma_{w}^{2} \leq \hat{\sigma_{w}^{2}} \leq\left(1+\varepsilon_{2}\right) \sigma_{w}^{2}, \\
& 0 \leq \varepsilon_{1}<1 ; \varepsilon_{2} \geq 0
\end{aligned}
$$

Let, $\quad \stackrel{\Delta}{=} \frac{1+\varepsilon_{2}}{1-\varepsilon_{1}} \geq 1$, where $U$ denotes the detection requirement of Probability of Detection $\left(\mathrm{P}_{\mathrm{d}}\right)$ and Probability of False Alarm $\left(\mathrm{P}_{\mathrm{f}}\right)$ and $\varepsilon 1$ and $\varepsilon 2$ denotes the minimum and data values for finding $P_{f}$ in primary and secondary users [7, 8]. The relation between observation window $\mathrm{N}$ and SNR is

$$
S N R=\frac{(U-1) \sqrt{N}+\sqrt{2} U Q^{-1}\left(P_{f}\right)-\sqrt{2} Q^{-1}\left(P_{d}\right)}{\sqrt{2} Q^{-1}\left(P_{d}\right)+\sqrt{N}}
$$

In the case for large observation window the relation can be writtenas:

$$
\begin{aligned}
& S N R=U-1+\frac{\sqrt{2} U Q^{-1}\left(P_{f}\right)-\sqrt{2} Q^{-1}\left(P_{d}\right)}{\sqrt{2} Q^{-1}\left(P_{d}\right)+\sqrt{N}} \\
& =U-1+O(\sqrt{1 / N}) \geq(S N R)_{\min } \\
& (S N R)_{\min } \stackrel{\Delta}{=}(U-1)
\end{aligned}
$$

In section II, we have implemented the test statistics of thesensed spectrumusing conventional Energy Detection technique, and the signals are taken from USRP. The 
conventional Welch's Cross PSD test statistics are modified by squaring the $\mathrm{M}^{\text {th }}$ segments of the Periodogram. In the section III of this paper,the proposed method is discussed where CWPS from an input signal obtainedafter sensing from NIUSRP-2920. The signal is applied through a low pass filter and resampled at $15 \mathrm{KHz}$. The interpolation and decimation factor are considered as 3 and 2 respectively while the gain is taken as $20 \mathrm{~dB}$. There after analyzing the PSD, the threshold has been set to $-80 \mathrm{~dB}$ and it is compared withthe obtained plot by using detection sensing technique. In the proposed case, SNR is considered as $10 \mathrm{~dB}$ in Rayleigh channel while noise variance in AWGN is taken as 0.5 . The sampling frequency is kept to $200 \mathrm{MHz}$ and number of samples from the filtered output is fixed to 2048 .

\section{Ber Calculation Based on Conventional Periodogram}

In our proposed method, the threshold is varied from $-75 \mathrm{~dB}$ to $-80 \mathrm{~dB}$ for analyzing the signal. It is observed that change in the value of threshold changes in error rate. Considering here the Binary FSK modulation technique, the BER isrepresented as [1]:

$$
P_{B E R}=Q\left(\sqrt{\frac{E_{b}}{N_{0}}}\right)=\frac{1}{2} \operatorname{erfc}\left(\sqrt{\frac{E_{b}}{2 N_{0}}}\right)
$$

where, $N_{0}$ denotes the noise variance and $\mathrm{E}_{\mathrm{s}}=\mathrm{E}_{\mathrm{b}} \log _{2} \mathrm{M}$ the symbol or total energy. $E_{b}$ is the bit per energy and $M$ constellation respectively. In the figure 1, it is shown that the error rates are getting changed for increasing the value of threshold for multiple channels. Thus averaging of threshold is increases in the error rates consistently. The threshold scale is taken in $\mathrm{x}=$ axis which corresponds 10 as $-75 \mathrm{~dB}$ and scaling the other values accordingly in the graph.

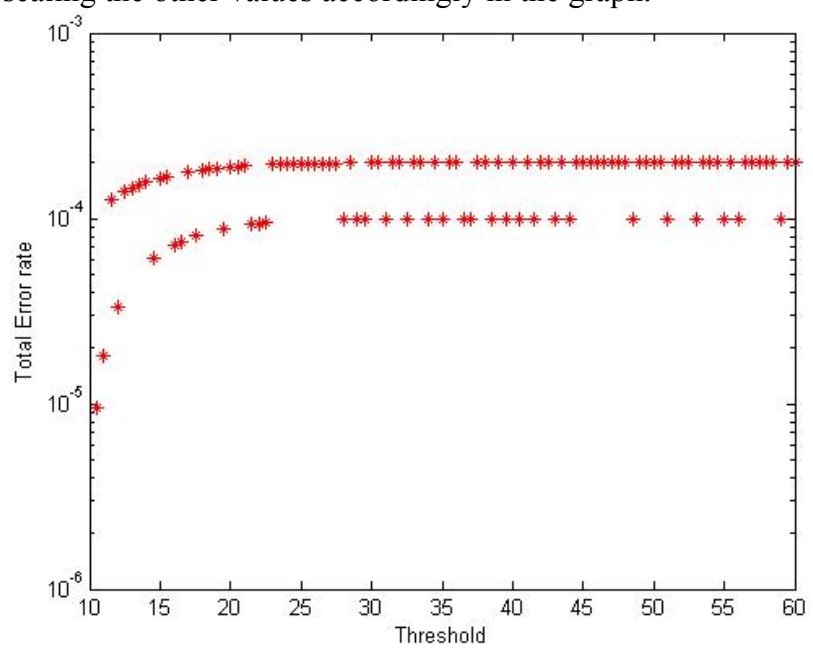

Fig 1. Total Error rate vs Threshold

Here,by taking 4000 elements of the obtainedPSD the $P_{d}$ is analyzed. In the Eq (8), the BER has been simulated with varying noise variance with constant input signal variance of 0.5 . So, for the addition of a random variable to the SNR the noise variance normalized [7]. A random variable is taken to be uniformly distributed in $[-1,1]$ which is added to the SNR input. The noise uncertainty becomes $\pm 1 \mathrm{~dB}$ (maximum) with respect to the signal variance.

\section{Analysis of BER Based on CWPS with Hamming Window}

Applying the Welch's PSD [2], the signal is splitted into 2048 overlapping segments of length 3999 samples. These overlapping segments are then windowed in the time domain $[8,9]$. The Periodogram equation after windowing the data segments can be written as:

$$
\stackrel{\vee}{X X}_{X X}^{i}(f)=\frac{1}{M U}\left|\sum_{x=0}^{M-1} X_{i}(n) w(n) e^{-j 2 \pi f n}\right|^{2} \quad l=0,1, \ldots . L-1
$$

where, $U$ is taken as a normalization factor for the power.Therefore $U$ can be written as:

$U=\frac{1}{M} \sum_{n=0}^{M-1} w^{2}(n)$

The Welch power spectrum estimate is simplified as:

$P_{X X}^{w}=\frac{1}{L} \sum_{i=0}^{L-1} P_{X X}^{\approx}(f)$

where, $P_{X X}$ represents the average of modified Periodogram.

The mean value of Welch estimate is denoted as:

$E\left[P_{X X}^{w}(f)\right]=\frac{1}{L} \sum_{i=0}^{L-1} E \tilde{P_{X X}^{i}}(f)$

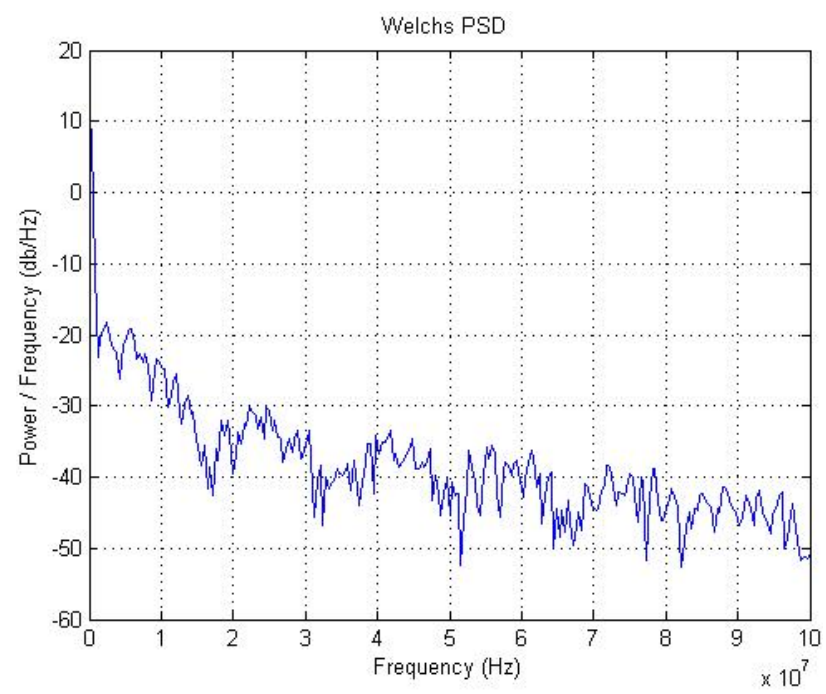

Fig 2. Welch's PSD plot with hamming window

The improved BER is calculated using the modified Welch's equation which is derived in the appendix. The comparison between the earlier BER in Fig 1 and Fig 3 depicts an improvement in the error rates. Thus by applying CWPS, the signal resolution is found to converge with respect to the constant phase and having any value from 0 to $90^{\circ}$ as taken from $\mathrm{Eq}(24)$ :

$B E R=\frac{1}{\pi} \int_{0}^{\pi / 2} \exp \left(-\frac{0.346}{\log _{2}^{M} G \sin \theta^{2}}\right) d \theta$ 


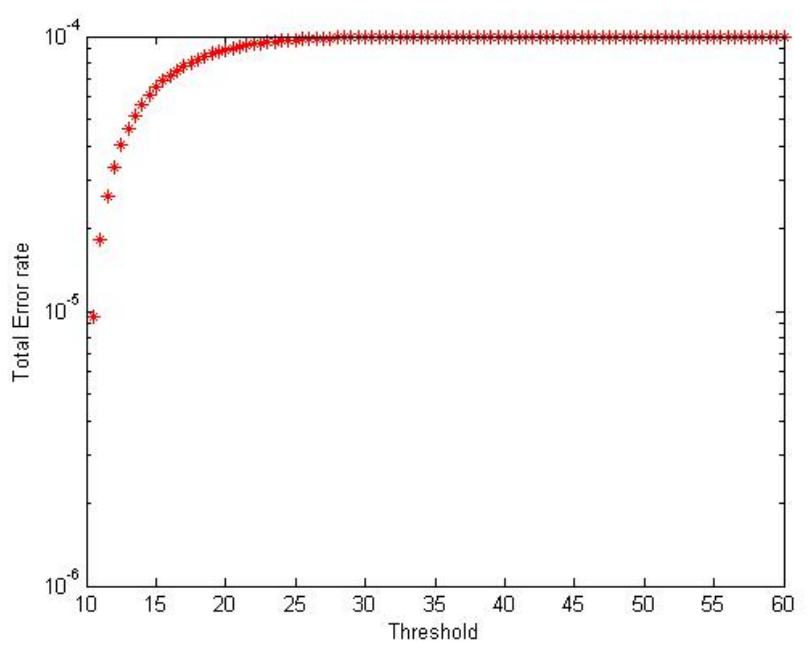

Fig 3. BER for Converged Welch's PSD

The modified hamming window is taken into account where windows are triangular in nature which helps in proper convergence in the resolution of the PSD. As shown in Fig 2, the plot of CWPS analysis shows a drastic improvement in PSD as compared with the earlier FFT/NFFT process output of the original signal. Therefore the corresponding BER having CWPS shows an improved error rate when compared to the conventional method.

\section{Conclusion}

An improved performance in BERis obtained using CWPS technique keeping SNR constant and varying threshold. For this novel CWPS approach a fixed effective radius of the network is considered and the symbol energy is assumed as total energy while evaluating the BER. Furthermore, the CWPS techniqueis compared to the modified Welch's conventional Periodogram at the time of analysis of BER. It is found that the nature of CWPS output is an improved version of earlier.This work can be further extended by performing the source detection and applying the same for cyclostationarity based applications in CR

\section{References}

1. S. Shellhammer, R. Tandra, 'Performance of the Power Detector with Noise Uncertainty', IEEE Std. 802.22-06/0134r0, (2006)

2. P. Welch, 'The use of fast Fourier transform for the estimation of power spectra: a method based on time averaging over short, modified periodograms', IEEE Transactions on Audio and Electroacoustics, vol. 15(2), pp.70-73, (1967)

3. Hansa R. Gupta, R.Mehra, 'Power Spectrum estimation using Welch Method for various Window Techniques', International Journal of Scientific Research Engineering \& Technology', vol. 2(6), pp. 389-392, (2013)

4. A. Mukherjee, et. al, "Source Number detection and Realization of ROC for Spectrum sensing in Cognitive Radio", Indian Journal of Science and Technology, vol. (27), DOI: 10.17485/ijst/2015/v8127/67727, pp. 1-5, 2015.

5. I. Harjula, A. Hekkala, M. Matinmikko, M. Mustonen, 'Performance evaluation of spectrum sensing using welch periodogram for OFDM signals', in Proceedings of the IEEE 73rd Vehicular Technology Conference, pp.1-5, (2011)
6. S. Han, S. Ahn, E. Oh, D. Hong, 'Effect of channel-estimation error on BER performance in cooperative transmission', IEEE Transactions onVehicular Technology, vol. 58(4), pp. 2083-2088, (2009)

7. A. Mukherjee et. al., "HML based Smart positioning of Fusion Center for Cooperative Communication in Cognitive Radio Networks", IEEE Communication Letters, vol. 20 (11), DOI: 10.1109/LCOMM.2016.2602266, 2016.

8. A. Mukherjee, et. al., "Spectrum sensing for cognitive radio using blind source separation and hidden Markov model", Fourth International Conference on Advanced Computing and Communication Technologies, pp. 409-414, DOI: 10.1109/ACCT.2014.63, 2014.

9. A. Mukherjee, et. al., "Optimization of sensing and detection in cognitive radio using energy based algorithm and quantized data fusion", International Conference for Convergence of Technology, pp. 362-368, 2014. 


\section{Appendix}

Derivation Of The Converged Welch's Power Spectral Algorithm

We know Welch's modified Periodogram[3] is written as:

$$
\stackrel{\vee}{P}_{X X}^{i}(f)=\frac{1}{M U}\left|\sum_{x=0}^{M-1} X_{i}(n) w(n) e^{-j 2 \pi f n}\right|^{2} \quad l=0,1, \ldots . L-1
$$

The input signal modulated by M-ary FSK modulation can be written as: $S_{M F S K}(t)=\sqrt{\frac{2 E_{s}}{T_{s}}} \cos \left(\frac{\pi}{T_{s}}\left(n_{c}+i\right) t\right)$

$=\sqrt{\frac{2 E_{s}}{T_{s}}} \cos \left(2 \pi\left(f_{c}+\frac{i}{2 T_{s}}\right) t\right), 0<t<T_{s}$

where, $f_{c}=n_{c} / 2 T_{s}$ denotes carrier frequency, and $n_{c}=$ the fixed integer. $E_{s}=E_{b} \log _{2}^{M} ; T_{s}=T_{b} \log _{2}^{M}$ the energy per symbol and symbol period respectively.

Now putting the value of $S_{\text {MFSK }}(t)$ in equation(21)in place of $x_{i}(n)$ it becomes:

$$
\stackrel{\vee}{P X}^{i}(f)=\frac{1}{M U}\left|\sum_{n=0}^{M-1} \sqrt{\frac{2 E_{s}}{T_{s}}} \cdot \cos \left(\frac{\pi}{T_{s}}\left(f_{c}+\frac{i}{2 T_{s}}\right) t\right) \cdot w(n) e^{-j 2 \pi f n}\right|^{2}
$$

Assuming $E_{s} \cong E_{\text {total }}$,

$$
E_{\text {total }}=\sum_{i=1}^{k} \sum_{t=1}^{n} E\left[\left|x_{i}, t\right|^{2}\right]
$$

$$
v^{i}
$$

$P_{x X}(f)=$

$\frac{1}{M U}\left|\sum_{n=0}^{M-1} \sqrt{\frac{2}{T_{s}} \sum_{i=1}^{k} \sum_{t=1}^{n} E\left[\left|x_{i}, t\right|^{2}\right]} \cdot \cos \left(\frac{\pi}{T_{s}}\left(f_{c}+\frac{i}{2 T_{s}}\right) t\right) \cdot w(n) e^{-j 2 \pi f n}\right|^{2}$

We know that, for a lower band, the minimum energy per bit for the described model is given by:

$$
E=\frac{N_{0} \log _{e}^{2}}{G}
$$

where, $\mathrm{G}$ denotes the effective radius of the network) Considering noise variance $N_{0}$ be 0.5 , the equation can be simplified as:

$$
\Rightarrow E=\frac{0.5 \log _{e}^{2}}{G}=\frac{0.346}{G}
$$

$$
v^{i}
$$$$
P_{X X}(f)=
$$

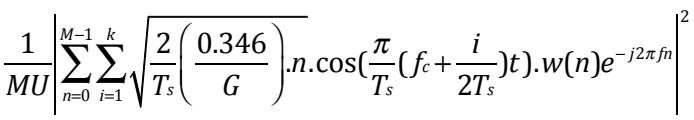

$v^{i}$

$P_{X X}(f)=$

$$
\begin{aligned}
& \frac{1}{M U}\left|\sum_{n=0}^{M-1} \sum_{i=1}^{k} \frac{0.831 \sqrt{n}}{\sqrt{T_{s} G}} \cdot \cos \left(\frac{\pi}{T_{s}}\left(f_{c}+\frac{i}{2 T_{s}}\right) t\right) \cdot w(n) e^{-j 2 \pi f n}\right|^{2} \\
& =\frac{0.831}{\sqrt{T_{s} G}} \cdot \frac{1}{M U}\left|\sum_{n=0}^{M-1} \sum_{i=1}^{k} \sqrt{n} \cdot \cos \left(\frac{\pi}{T_{s}}\left(f_{c}+\frac{i}{2 T_{s}}\right) t\right) \cdot w(n) e^{-j 2 \pi f n}\right|^{2}
\end{aligned}
$$

Assuming this $\sqrt{T_{s} G}$ is coefficient of converging and as $T_{s}\left(T_{s}=T_{b} \log _{2}^{M}\right)$ increases the $\mathrm{W}_{\mathrm{PSD}}$ resolution decreases keeping energy per symbol constant.

$\because T_{s} \propto T_{b}$ and if we consider the power per bit as $P_{b}$, then $P_{b}$ $T_{b}=E_{b}$

$\therefore T_{b} \propto \frac{E_{b}}{P_{b}}$ and $T_{s} \propto \frac{E_{s}}{P_{s}} P_{s}$ is the power per symbol. On the

other hand it can be observed that BER of the signal improves asfollowed in the below derivation. In BFSK modulation technique, BER is calculated as:

$B E R=\frac{1}{2} \operatorname{erfc}\left(\sqrt{E_{b} / 2 N_{0}}\right)$

where, $\operatorname{erfc}(x)=\frac{2}{\pi} \int_{0}^{\pi / 2} \exp \left(-\frac{x^{2}}{\sin ^{2} \theta}\right) d \theta$

$\therefore B E R=\frac{1}{2} \cdot \frac{2}{\pi} \int_{0}^{\pi / 2} \exp \left(-\frac{E_{b} / 2 N_{0}}{\sin ^{2} \theta}\right) d \theta$

As we have previously assumed that $E_{s} \cong E_{\text {total }}$ and $E=\frac{0.346}{G}$, so from $E_{s}=E_{b} \log _{2}^{M}$ the $B E R$ equation can be simplified as:

$B E R=\frac{1}{\pi} \int_{0}^{\pi / 2} \exp \left(-\frac{0.346}{\log _{2}^{M} G \sin \theta^{2}}\right) d \theta$

Now, if we compare the derived BER equation with error function then it will be seen that the value of $x$ in $\operatorname{erfc}(x)$ is

$\sqrt{-\frac{0.346}{\log _{2}^{M} G \sin \theta^{2}}}$

i.e. $\frac{E_{b}}{2 N_{0}}=\sqrt{\frac{0.346}{\log _{2}^{M} G}}=\frac{0.588}{\sqrt{\log _{2}^{M}} \cdot \sqrt{G}}$ 